\title{
High rates of culture conversion and low loss to follow-up in MDR-TB patients managed at Regional Referral Hospitals in Uganda
}

\author{
Martin Kayitale Mbonye ( $\square$ mbonyemarti@yahoo.com ) \\ 9538 \\ John-Paul Otuba \\ University Research Co., LLC

\section{Sara Riese} \\ University Research Co., LLC \\ Hilary Alima \\ University Research Co., LLC \\ Frank Mugabe \\ National TB and Leprosy Programme, Ministry of Health \\ Augustin K Muhwezi \\ University Research Co., LLC

\section{Stavia Turyahabwe} \\ National TB and Leprosy Programme, Ministry of Health

\section{Christopher Wandera} \\ University Research Co., LLC \\ Tisna Veldhuijzen van Zanten \\ University Research Co., LLC

\section{Gladys Tugume} \\ University Research Co., LLC
}

Makerere University College of Business and Management Sciences https://orcid.org/0000-0002-9103-

\section{Research article}

Keywords: Multi-drug resistant tuberculosis, regional referral hospitals, Uganda

Posted Date: May 30th, 2019

DOI: https://doi.org/10.21203/rs.2.9187/v2

License: (c) (1) This work is licensed under a Creative Commons Attribution 4.0 International License. Read Full License 


\section{Abstract}

Background: Multi-drug resistant - tuberculosis (MDR-TB) is an emerging public health concern in Uganda. Prior to 2013, MDR-TB treatment in Uganda was only provided at the national referral hospital and two private-not-for profit clinics. From 2013, it was scaled up to seven regional referral hospitals (RRH). The aim of this study was to measure interim (six months) treatment outcomes among the first cohort of patients started on MDR-TB treatment at the RRH in Uganda. Methods: This was a cross-sectional study in which a retrospective descriptive analysis of data on a cohort of 69 patients started on MDR-TB treatment at 7 RRH between 1st April 2013 and 30th June 2014 and had been on treatment for at least nine months was conducted. Results: Of the 69 patients, 21 (30.4\%) were female, 39 (56.5\%) HIV-negative, 30 (43.5\%) resistant to both isoniazid and rifampicin and $57(82.6 \%)$ category 1 or 2 drug susceptible TB treatment failures. Median age at start of treatment was 35 years (Interquartile range (IQR): 27-45), median time-to-treatment initiation was 27.5 (IQR:6-89) days and of the 30 HIV-positive patients, 27 (90.0\%) were on anti-retroviral treatment with a median CD4 count of 206 cells/microliter of blood (IQR: 113-364.5). Within six months of treatment, $59(86.0 \%)$ patients culture converted, of which $45(65.2 \%)$ converted by the second month and the other $14(20.3 \%)$ by the sixth month; one (1.5\%) did not culture convert; three $(4.4 \%)$ died; and six $(8.8 \%)$ were lost-to-follow up. Fifty (76.8\%) patients experienced at least one drug adverse event, while $40(67.8 \%)$ gained weight. Mean weight gained was 4.7 (standard deviation:3.2) kilograms. Conclusions: Despite MDR-TB treatment initiation delays, most patients had favourable interim treatment outcomes with majority culture converting early and very few getting lost to follow-up. These encouraging interim outcomes indicate a successful scale-up of MDR-TB treatment to RRH.

\section{Background}

The diagnosis and treatment of Multidrug Resistant Tuberculosis (MDR-TB) remains a global challenge (1). MDR-TB is defined as a form of Tuberculosis (TB) infection that is caused by bacteria that are resistant to two of the most efficacious first line anti-TB medicines (Isoniazid and Rifampicin) (2). According to the World Health Organization (WHO), there was an estimated 558,00 new cases of MDR-TB of which only 160,684 cases were diagnosed in 2017 (3). Globally, the treatment outcomes of MDR-TB are less favorable, with only a $50 \%$ treatment success rate (3), partly due the long duration of treatment, extending up to 24 months, treatment adherence difficulties and common adverse events $(2,4)$. Although WHO has been very supportive in ensuring availability of appropriate guidelines for diagnosis and treatment of MDR-TB (2,5-7), access to MDR-TB treatment in high burden countries has been increasing but at a less than desired pace (8) indicating challenges in implementation (9). Until recently, the limited availability of second line drugs for the treatment of MDR-TB has been a major barrier to treatment access (10). The gap in MDR-TB treatment coverage globally has also been attributed to inadequacies within the health system, including but not limited to lack of skilled human resources for health, lack of MDR-TB medicines and other logistics for MDR-TB patient management, inadequate infrastructure for in-patient care and suboptimal TB infection control practices, lack of funding for patient social support and limited access to laboratory and other monitoring tests (2).

As of 2014, Uganda was one of the 22 high burden TB countries in the world with approximately 44,187 incident TB cases in 2014 (161/100,000). Additionally, 50 - 60\% of TB patients are co-infected with HIV, and 
the country suffers a generalised HIV epidemic with a national prevalence of $7.3 \%$ (11). It is estimated that approximately $1.4 \%$ of new sputum positive cases and $12.1 \%$ of previously treated TB cases were MDR-TB, which translated into approximately 1,100 new MDR-TB cases in 2014 alone (3). However, the MDR-TB case notification rate was notably much higher than the MDR-TB treatment enrollment rate (12), indicating gaps in access to MDR-TB treatment. In order to improve access to MDR-TB treatment, it was necessary to further decentralise MDR-TB services. Until 2012, MDR-TB treatment was only being provided at the National Referral Hospital (NRH) and two Médecins Sans Frontières (MSF) run clinics at Arua Regional Referral Hospital (RRH) and Kitgum government general hospital (13). In 2012, the National TB and Leprosy Programme (NTLP) began mobilizing partner support to decentralize treatment of drug resistant TB (DR-TB) from National Referral and two Medicines Sans Frontiers clinics to the regional referral hospitals (RRH) to increase coverage and make treatment more accessible.

In collaboration with NTLP, the United States Agency for International Development (USAID) funded Strengthening Uganda's System for Treating AIDS Nationally (SUSTAIN) project embarked on capacity building activities to manage drug resistant TB at seven RRH in 2013. A phased approach to scale up ambulatory MDR-TB care to the seven RRH with the highest incidences of MDR-TB was adopted. Scale-up included training of multi-disciplinary teams at each RRH to initiate and manage MDR-TB treatment, dissemination of guidelines for management of MDR-TB, and facilitation of follow-up of patients and their household contacts. The multidisciplinary teams were hospital staff involved in providing varying services to MDR-TB patients and comprised: members of the clinical team including, an audiologist and nurses; members of laboratory teams including laboratory technologists, technicians and assistants; the medical social workers including counsellors; a psychiatrist; and at least one member of the hospital administration.

WHO recommends MDR-TB models that are ambulatory, in which patients receive medicines from a local health facility close to their homes, or have the medicines delivered daily by a health worker to the patient's home, as compared to hospital-based models of care in which patients are admitted into a centralized setting for an intensive phase of treatment or more, lasting a minimum of six months $(2,14)$. In Uganda, the NTLP recommends a mixed model of care that combines both ambulatory and hospitalisation approaches (15).

To understand if scale up of MDR-TB treatment to RRH was successful in ensuring favorable interim treatment outcomes (sputum culture conversion), we conducted a retrospective analysis for the early treatment outcomes for the first cohort of MDR-TB patients started on MDR-TB treatment at seven RRH. To our knowledge, this is the first analysis of this kind in the country.

\section{Methods}

This was a cross-section study in which clinical records of 69 patients started on MDR-TB treatment at seven RRHs between $1^{\text {st }}$ April 2013 and $30^{\text {th }}$ June 2014 were retrospectively examined. At the time of the review, each patient had been on treatment for at least nine months and their six-month culture results were available. The patients whose records were reviewed included patients diagnosed with MDR-TB using culture based drug susceptibility testing (DST) or rifampicin resistance (RR) established through rapid test Xpert ${ }^{\circledR}$ MTB/RIF. 


\section{Mycobacteria Culture and Drug Susceptibility Testing}

Prior to the study period, laboratories of the seven RRH collected sputum samples and delivered them to the National TB Reference Laboratory (NTRL) for culture and drug sensitivity testing. Samples were from presumptive MDR-TB cases, defined as patients that: 1) failed on category 1 (patients that were previously undergoing pulmonary bacteriologically confirmed TB treatment for the first time and were sputum smearpositive at 5 months or later during the course of treatment) or category 2 (patients that were previously undergoing retreatment for pulmonary bacteriologically confirmed TB and were sputum smear-positive at 3 months or later during the course of treatment); 2) relapsed (previously treated and cured of TB but then returned with a pulmonary bacteriologically confirmed TB); 3) returned after loss to follow-up (did not start the previous TB treatment or treatment was interrupted for two or more months); or 4) were household contacts of confirmed MDR-TB cases (16).

The NTRL conducted cultures on BACTEC MGIT 960 or Lowenstein-Jensen solid medium, while conventional DST or Line Probe Assays (LPA), a rapid molecular method for detecting first and second line drug resistance were used. The MOH/NTLP adopted and installed GeneXpert MTB/RIF machines in 2011 to enable them conduct rapid DST for screening for Rifampicin Resistance (RR), a proxy for MDR-TB. All cases of RR had sputum samples referred to the NTRL for baseline culture prior to treatment initiation $(5,15,16)$.

\section{MDR-TB Regimen and Treatment Follow-up}

Baseline history, physical examination, chest radiography, haematological and chemistry tests were conducted at the RRH before initiating a patient on MDR-TB treatment. Multidisciplinary teams conducted pre-treatment preparation and counselling, after which a standardised MDR-TB regimen was initiated in accordance with Uganda National MDR-TB guidelines (15). The standardised MDR-TB regimen consisted of five drugs; 1) one injectable agent including either Kanamycin or Capreomycin or Amikacin substituted depending on need, 2) a fluoroquinolone (levofloxacin), 3) Pyrazinamide, 4) Ethionamide and 5) Cycloserin. Para-amino salicylic acid (PAS) was also available for substitution if Cycloserin was not tolerated. Both adult and child contacts of MDR-TB patients that were symptomatic but tested negative for MDR-TB would be treated with the regimen of the index patient (empirically). All treatment was administered daily through Directly Observed Therapy (DOT), except the injectable that was given for only six days in a week (15).

Patients were initiated on an ambulatory or hospitalisation model depending on severity of the illness. The very sick patients were initiated on a hospitalization model and when they stabilised on treatment, they chose a peripheral health facility nearest to their place of abode where to receive their treatment from (ambulatory model). The patient was then transferred to the peripheral health facility to continue receiving ambulatory DOT, adherence monitoring and psychosocial support. Before transferring the patient to the peripheral health facility, a team of health workers from the RRH visited the health facility and assessed it and also trained staff in MDR-TB case management. The initiating RRH conducted monthly visits to the peripheral sites and provided supervision and mentorship to health workers. A monthly supply of MDR-TB and ancillary 
medicines and personal protective equipment for TB infection control were also delivered to the health facility.

The treatment for all MDR-TB patients involved two phases, the intensive phase and continuation phase. During the intensive phase, the patient received an injectable agent described above until they culture converted Cultures were done before the start of treatment and subsequently every other month. Culture conversion was defined as two consecutive negative cultures, thirty days apart after commencing treatment. After culture conversion, the patient continued on the injectable agent for an additional four months and thereafter started the continuation phase up to the eighteenth $\left(18^{\text {th }}\right)$ month (15). During the intensive phase, all MDR-TB patients were counselled and tested for HIV. MDR-TB/HIV co-infected patients that were not on anti-retroviral treatment (ART) already were linked to integrated TB/HIV services and initiated on an ART regimen containing a backbone of either Zidovudine/Lamivudine or Tenofovir/Emtricitabine and a tail of Efavirenz or Nevirapine within two months of MDR-TB treatment initiation, regardless of CD4+ count (17). Also, during this phase, health workers conducted home visits to provide relevant health education to the patient and family, assess for adherence to infection control practices, screen household contacts for TB symptoms and collect sputum samples for GeneXpert® MTB/RIF testing.

At the $\mathrm{RRH}$, the patients received a comprehensive monthly review in which sputum samples were collected for monthly follow-up cultures, kidney and liver functions were analysed, blood samples were collected and delivered to a private laboratory for thyroid function tests and patients were assessed for hearing impairment. For co-infected MDR-TB/HIV patients, all services integrated MDR-TB and HIV care, such as screening and management of opportunistic infections, response to ART, Immune Reconstitution Inflammatory Syndrome, repeat CD4+ and Viral load testing after 6 months according to WHO HIV treatment guidelines (17). Also, during the monthly review, the patients received nutritional assessment, counselling and support.

All confirmed MDR-TB patients provided sputum samples for smears and baseline DST before treatment initiation. Subsequently, sputum was collected and tested every month during the twenty-four months of treatment.

During the treatment, patients were monitored for drug adverse events and managed with dose adjustments, substitution of the offending drug, treatment withdrawal and/or by reassurance. Adverse events were either self-reported or clinically confirmed through conducting various physical and laboratory examinations.

\section{Data collection and analysis}

Demographic data, previous TB treatment history, HIV status and previous exposure to ART, CD4+ count, drug resistance patterns, MDR-TB treatment regimen, time to treatment initiation, monthly weights, occurance of adverse events, monthly smear and culture monitoring were collected.

The primary treatment outcome was the proportion of patients that culture converted at 6 months of treatment. Culture convertion is considered a useful indicator of MDR-TB programme effectiveness $(2,18)$. Culture conversion has been defined above as two consecutive negative cultures taken one month apart 
following commencement of MDR-TB treatment (18). Secondary outcomes included time to culture conversion - time from treatment initiation to the date of the first of two consecutive negative cultures (6). Univariate analysis (Chi-square test) were perfomed to determine the associations between type of patient and treatment outcome. The type of patient was categorised as: new patient (no previous history of TB infection), relapse (a patient who previously had TB infection, completed treatment and was cured but has got TB infection again), defaulter (a patient who previously had TB but defaulted on treatment) and treatment failiure (a patient who previously had TB infection, completed treatment but was not cured). STATA statistical sofware (version 14.0) was used for all the analysis.

\section{Results}

\section{Demographic and Clinical Characteristics}

A total of 73 patients were started on MDR-TB treatment from $1^{\text {st }}$ April 2013 through $30^{\text {th }}$ June 2014 (Figure 1). Four patients started on an empiric MDR-TB treatment regimen were excluded from the analysis because two of the patients were found without MDR-TB following a culture andDrug Susceptibility Testing (DST), while the other two were culture negative. The tests were carried out at the National TB Reference Laboratory.

Baseline characteristics of the 69 patients whose data was analysed are shown in Table 1. Twenty-one (30.4\%) patients were female, median age was 35 (Inter Quartile range (IQR): 27 - 25) years. Median time to MDR-TB treatment initiation was 27.5 days (IQR: 6 - 89). The drug susceptibility patterns were as follows: a total of $30(43.5 \%)$ patients were resistant to both isoniazid and rifampicin on culture based DST and 39 (56.5\%) on MTB/RIF based on GeneXpert ${ }^{\circledR}$ result. A total of 66 (95.6\%) patients were on a standardised treatment regimen. Thirty (43.5\%) patients were HIV-positive, of which which $27(90.0 \%)$ were already receiving anti retroviral treatment (ART), with median CD4 count of 238 cells/microliter of blood (IQR214 to 307). The proportion of HIV positive patients was higher among males (60.4\%) than females $(47.6 \%)$ by 12.8 percentage points. Additionally, the median CD 4 cell count was higher among males ( 268 cells/microliter of

blood) than femlaes ( 130 cells/microliter of blood). At the start of the MDR-TB treament five $(7.3 \%)$ patients did not have a history of previous TB treatment while twenty (29.0\%) had been Category 1 failures and 37 (53.6\%) Category 2 failures, one (1.5\%) was a relapse case and six (8.7\%) were returning lost to follow-up cases. Of the 59 patients that had their weight taken at both baseline and after six months of treatment, 40 (67.8\%) patients gained weight (mean weight gain: 4.7 kilograms; SD: \pm 3.2 ), one maintained the same weight while the other eighteen (30.5\%) lost weight (mean weight lost: 2.8 kilograms and SD \pm 2.3 ).

\section{Adverse Events}

Table 1 shows that 53 (76.8\%) of the patients experienced some form of adverse event associated with MDRTB medicines. Table 2 shows the specific MDR-TB associated adverse events that were experienced by patients. The most prevalent adverse event was hearing impairment (30.4\%). With exception of burning pains in the lower limbs, adverse events were managed symptomatically without need for treatment discontinuation.

Interim Treatment Outcomes 
Table 3 shows the six months interim treatment outcomes and the period to culture conversion. A total of 59 (85.5\%) of the 69 patients culture converted within a period of six months. Of the 59 patients with favourable treatment outcomes, $45(79 \%)$ culture convered within 2 months of treatment initiation and the remaining 14 $(21 \%)$ within the other 4 months. Six months after starting treatment ten patients had not achieved favourable treatment outcomes. Of these, three (4.4\%) patients had died, three (4.4\%) patients were lost to follow up, culture results were unknown for three (4.4\%) patients, while one patient did not culture convert. Figure 2 also shows the month by which each of the 59 patients with favourable treatment outcomes culture converted. Of the 59 patients that culture converted, 57 (96.6\%) achieved culture conversion witn 3 months following onset of treatment. The mean time to culture conversion was 1.98 (SD: \pm 0.86$)$ months (Table 3 ).

\section{Relationship between patient category and treatment outcome}

Table 4 shows that $86.4 \%$ of the MDR-TB patients with favourable outcomes (had sputum culture conversion) had been treatment failures on $1^{\text {st }}$ line (susceptible TB) treatment, compared to $60 \%$ of MDR-TB patients with unfavorable outcomes (did not culture convert, or lost to follow-up). Overall, there was a statistically significant association between patient type and treatment outcomes (Chi-square $=8.5$, and $\mathrm{p}$ value $=0.037$ ). Table 4 shows significant associations between patient type and six-month treatment outcomes. It was observed that patients who were failures on $1^{\text {st }}$ line treatment were less likely to experience unfavorable treatment outcomes by six months of MDR-TB treatment compared to defaulters, new and relapse patients $(p=0.04)$.

\section{Discussion}

This manuscript describes the first cohort of patients treated for MDR-TB at the RRH following scale up toRRH in Uganda. Overall, $87 \%$ of the patients had a favourable treatment outcomes, that is they culture converted within six months of onset of MDR-TB treatment.

There is generally paucity of literature on MDR-TB treatment outcomes among cohorts on treatment on the African continent $(19,20)$ although some cohorts have been described in South Africa, Lesotho, Nigeria and Tanzania (21-25). Our findings showed an exceptionally high sputum culture convertion rate (87\%), and these results were comparable to other studies done elsewhere (70\%) in the following 5 countries: Estonia, Latvia, Peru, the Philippines, and the Russian Federation (26).

The $4.4 \%$ loss to follow up rate in observed in this patient population is low compared to previous community based cohorts $(27,28)$. We postulate that the MDR-TB treatment model that adopted combining hospitalization model for sick patients and ambulatory model when patients were better ensured patients received treatment easily either during hospitalization or needed to travel only a short distance to receive treatment at the peripheral health facility. During the treatment patients were also able to interact more with trained healthworkers who re-enforced adherence and retention in care. The decentralisation of MDR-TB management to $\mathrm{RRH}$, coupled with utilisation of periheral health facilities nearest to patient homes for daily directly observed therapy (DOT) broadened MDR-TB treatment coverage and access and miminised loss to follow up. 
We observed a high number of patients (76.8\%) experienced some sort of drug adverse events. Adverse events like hearing impairment were majorly due to the MDR-TB medicines in the treatment regimen, like Kanamycin. Routine audiometry and Kanamycin substitution may therefore benefit the patient. Other adverse events like peripheral neuropathy are known pyrazinamide,ethionamide and cycloserine associated events, potentiated by HIV related peripheral neuropathies. We postulate that the high frequency of adverse events observed was probably associated with poor clinical condition of the patients and existing MDR-TB/HIV coinfection. Further research is needed to elucidate predictors of adverse events among MDR-TB patients on treatment.

Generally, mortality was low (4.4\%) occurring in only three patients - all HIV co-infected. This trend in mortality among HIV co-infected patients compares with observations in previous studies (18). In our study, mortality occurred early-within 4 weeks of MDR-TB treatment initiation, underscoring the need for expedited treatment initiation among MDR-TB patients. A meta-analysis by Wells et al. reported death among MDR-TB patients on treatment occuring within 4-8 weeks of MDR-TB diagnosis and also demonstrated higher mortality rates among HIV co-infected patients, and even among patients on ART (29). Interestingly, the majority of the patients were HIV-negative, while most of the HIV-positive patients were on ART. Further studies need to be conducted to identify factors contributing to a higher MDR-TB prevalence among HIV seronegative patients. We postulate that high prevelence of HIV seronegative patients may have been as a result of MDR-TB/HIV co-infected patients dying during the long time-to-treatment initiation periods, leaving the HIV seronegative MDR-TB patients.

Until 2012, MDR-TB treatment in Uganda was only provided at the National Referral Hospital (NRH) and two being Médecins Sans Frontières (MSF) run MDR-TB clinics at Arua RRH and Kitgum government general hospital (13). At that time, RRH only had capacity to do surveillance and referral to the three sites for treatment. Patients diagnosed at RRH had challenges accessing treatment, due to long distances to the centers, lack of social/family support and lack of food packages during the mandatory hospitalization phase of treatment. The SUSTAIN project supported 7 RRH's MDR-TB treatment sites tried to address the access and coverage challenges since 2013. The early treatment outcomes from our study indicate a successful scale up of MDR-TB treatment to RRH.

We attribute these outcomes primarily to six aspects of the MDR-TB treatment scale-up; first, to utilization of an effective - standardized MDR-TB treatment regimen that made training, drug forecasting and management of drugs adverse events simpler; Second, to the existing DOT at peripheral health facilities supervised by the decentralized RRH MDR-TB sites which enabled individual patients' access daily medication from facilities closest to their homes as compared to having the DOT site at the RRH and NRH; Third, to the monthly reviews of patients by the multidisciplinary teams, that aided prompt identification and management of adverse events minimizing treatment interruptions and ultimately loss to follow-up; Fourth, to the monthly home visits and psychosocial support, adherence counselling and nutritional assessment counselling and support provide by the RRH multidisciplinary team; Fifth, the integrated MDR-TB/HIV services run by the MDR-TB clinics, which reduced patient waiting time and also provided an opportunity to the multidisciplinary teams to screen for drug adverse events and interactions, and provide appropriate management. Finally, we think that 
the ambulatory care coupled with TB infection control measures and practices at the RRH during the early days of hospitalization may have contributed to prevention of reinfection with resistant strains.

The feasibility of MDR-TB models of care delivered through ambulatories is still debated $(23,24,30)$. Equally, community based cohorts have demonstrated challenges with patient adherence, leading to high lost to follow-up rates $(27,28)$. Our findings provide insights into the early outcomes of the mixed model of MDR-TB care in resource constrained settings. Adopting both models of care provides the best opportunity to identify patients at higher risk of loss to follow-up.

The study was not without limitations. The source of data patient registers since they were available at the health facilities. Using data from patient medical note books would have enabled to obtain more data on results of clinical examination to futher describe patient profiles. The clinics were also not capturing data on patient's height and thus it was not possible to measure important antopometric indicators such as Body Mass Index to explain nutritional status of patients. Lastly, this study was not designed to find if a mixed model of care is superior to single dimensional models. However, the results point towards early success of the programme. We believe that delivering a decentralized MDR-TB care through RRH with utilization of peripheral DOT health facilities is feasible and could serve for resource limited settings. The final treatment outcomes needs to be evaluated as we push for the need for rapid scale up of coverage and access to MDRTB care to lower level health facilities using this model. Further, qualitative studies are necessary to provide insights into health workers' perspectives and patient's experiences regarding the mixed model of MDR-TB care in Uganda.

\section{List Of Abbreviations}

ART Anti-Retroviral Therapy

CHC Center for Health Services

DOT Directly Observed Treatment

DST Drug Susceptibility Test

HIV Human Immuno-Deficiency Virus

LPA Line Probe Assays

MDR Multi-Drug Resistance Tuberculosis

MSF Médecins Sans Frontières

NTLP National TB and Leprosy Program

NTRL National TB Reference Laboratory

PAS Para-Amino Salicylic Acid 
REC Research and Ethics Committee

RR Rifampicin Resistance

RRH Regional Referral Hospital

SD Standard Deviation

SUSTAIN Strengthening Uganda Health Systems for Treating AIDS Nationally

TB Tuberculosis

URC University Research Co., LLC

USAID United States Agency for International Development

WHO World Health Organization

\section{Declarations}

\section{Ethics approval and consent to participate}

Ethical approval was granted by the University Research Co., LLC | Center for Human Services Research and Ethics Committee (URC-CHS REC), Mildmay Uganda Research and Ethics committee (MUREC) and the Uganda National Council of Science and Technology (UNCST). MUREC waived the need for informed patient consent as the study only involved routinely collected patient monitoring data.

\section{Consent for publication}

Not applicable

\section{Availability of data and materials}

The dataset used during this study is available and can be shared upon request from the corresponding author.

\section{Competing Interests}

All authors declare they have no competing interests.

\section{Funding}


The SUSTAIN project was funded by the United States Agency for International Development (USAID) under Cooperative Agreement number 617-A-10-00007-00.

\section{Authors' contributions}

MKM, OJP, AH, MKA, WC, GT and KSW conceived and designed the study. MKM and OJP wrote study protocol. OJP and WC collected the data. MKM, OJP and WC electronically captured the data and performed data cleaning. MKM analyzed the data: MKM and OJP interpreted the results and wrote the manuscript. MKM, OJP and SR reviewed the manuscript to ensure it meets the submission requirements: All authors reviewed the manuscript and approved it for final submission.

\section{Acknowledgments}

We are grateful to USAID for funding the SUSTAIN project. We are also grateful for the support provided by the Ministry of Health and management of the Regional Referral Hospitals that participated in this study. We thank the healthcare providers who treat the MDR-TB patients at the study hospitals for supporting the data collection process. We would also like to thank Beth Turesson and Chana Rabiner who did the initial review of this manuscript.

\section{References}

1. Gandhi NR, Nunn P, Dheda K, Schaaf HS, Zignol M, Van Soolingen D, et al. Multidrug-resistant and extensively drug-resistant tuberculosis: a threat to global control of tuberculosis. The Lancet. 2010;375(9728):1830-43.

2. WHO. Guidelines for the programmatic management of drug-resistant tuberculosis-2011 update. Geneva: World Health Organization; 2011.

3. WHO. Global Tuberculosis Report. Geneva: World Health Organization. 2018.

4. Shenoi S, Heysell S, Moll A, Friedland G. Multidrug-resistant and extensively drug-resistant tuberculosis: consequences for the global HIV community. Current opinion in infectious diseases. 2009;22(1):11.

5. WHO. Companion handbook to the WHO guidelines for the programmatic management of drug-resistant tuberculosis: World Health Organization; 2014.

6. WHO. Treatment of tuberculosis: guidelines 4th Edition. 4th Edition ed: World Health Organization and Stop TB Initiative; 2010.

7. WHO. WHO consolidated guidelines on drug-resistant tuberculosis treatment. 2019.

8. Falzon D, Mirzayev F, Wares F, Baena IG, Zignol M, Linh N, et al. Multidrug-resistant tuberculosis around the world: what progress has been made? European Respiratory Journal. 2015;45(1):150-60. 
9. Du Cros P, Swaminathan A, Bobokhojaev O, Sharifovna Z, Martin C, Herboczek K, et al. Challenges and solutions to implementing drug-resistant tuberculosis programmes for children in Central Asia. Public health action. 2015;5(2):99-102.

10. Lunte K, Cordier-Lassalle T, Keravec J. Reducing the price of treatment for multidrug-resistant tuberculosis through the Global Drug Facility. Bulletin of the World Health Organization. 2015;93:279-82.

11. MOH, ICF. Uganda AIDS indicator survey 2011: UgandaMinistry of Health, ICF International; 2012.

12. WHO. Global Tuberculosis Report. Geneva: World Health Organization. 2015.

13. Luyirika E, Nsobya H, Batamwita R, Busingye P, Musoke W, Nabiddo L, et al. A home-based approach to managing multi-drug resistant tuberculosis in Uganda: a case report. AIDS research and therapy. 2012;9(1):12.

14. WHO. Multidrug and extensively drug-resistant TB (M/XDR-TB): 2010 global report on surveillance and response. Multidrug and extensively drug-resistant TB (M/XDR-TB): 2010 global report on surveillance and response. 2010.

15. MOH. National Guidelines for the Programmatic Management of Drug Resistant-TB. Kampala, Uganda: Uganda Ministry of Health; 2015.

16. $\mathrm{MOH}$. National Tuberculosis and Leprosy Programme Manual, 2nd edition. Kampala, Uganda: Uganda Ministry of Health; 2010.

17. WHO. March 2014 supplement to the 2013 consolidated guidelines on the use of antiretroviral drugs for treating and preventing HIV infection: recommendations for a public health approach. 2014.

18. Laserson KF, Thorpe L, Leimane V, Weyer K, Mitnick C, Riekstina V, et al. Speaking the same language: treatment outcome definitions for multidrug-resistant tuberculosis. The International Journal of Tuberculosis and Lung Disease. 2005;9(6):640-5.

19. Ahuja SD, Ashkin D, Avendano M, Banerjee R, Bauer M, Bayona JN, et al. Multidrug resistant pulmonary tuberculosis treatment regimens and patient outcomes: an individual patient data meta-analysis of 9,153 patients. PLoS medicine. 2012;9(8):e1001300.

20. Johnston JC, Shahidi NC, Sadatsafavi M, Fitzgerald JM. Treatment outcomes of multidrug-resistant tuberculosis: a systematic review and meta-analysis. PloS one. 2009;4(9):e6914.

21. Lalloo UG, Naidoo R, Ambaram A. Recent advances in the medical and surgical treatment of multi-drug resistant tuberculosis. Current opinion in pulmonary medicine. 2006;12(3):179-85.

22. Loveday M, Wallengren K, Voce A, Margot B, Reddy T, Master I, et al. Comparing early treatment outcomes of MDR-TB in decentralized and centralized settings in KwaZulu-Natal, South Africa. The international journal of tuberculosis and lung disease. 2012;16(2):209-15. 
23. Seung KJ, Omatayo DB, Keshavjee S, Furin JJ, Farmer PE, Satti H. Early outcomes of MDR-TB treatment in a high HIV-prevalence setting in Southern Africa. PloS one. 2009;4(9):e7186.

24. Oladimeji O, Isaakidis P, Obasanya OJ, Eltayeb O, Khogali M, Van den Bergh R, et al. Intensive-phase treatment outcomes among hospitalized multidrug-resistant tuberculosis patients: results from a nationwide cohort in Nigeria. PloS one. 2014;9(4):e94393.

25. Mpagama SG, Heysell SK, Ndusilo ND, Kumburu HH, Lekule IA, Kisonga RM, et al. Diagnosis and interim treatment outcomes from the first cohort of multidrug-resistant tuberculosis patients in Tanzania. PloS one. 2013;8(5):e62034.

26. Nathanson E, Lambregts-van Weezenbeek C, Rich ML, Gupta R, Bayona J, Blöndal K, et al. Multidrugresistant tuberculosis management in resource-limited settings. Emerging infectious diseases. 2006;12(9):1389.

27. Mitnick C, Bayona J, Palacios E, Shin S, Furin J, Alcántara F, et al. Community-based therapy for multidrug-resistant tuberculosis in Lima, Peru. New England Journal of Medicine. 2003;348(2):119-28.

28. Heller T, Lessells R, Wallrauch C, Bärnighausen T, Cooke G, Mhlongo L, et al. Community-based treatment for multidrug-resistant tuberculosis in rural KwaZulu-Natal, South Africa. The international journal of tuberculosis and lung disease. 2010;14(4):420-6.

29. Wells CD, Cegielski JP, Nelson LJ, Laserson KF, Holtz TH, Finlay A, et al. HIV infection and multidrugresistant tuberculosis-the perfect storm. The Journal of infectious diseases. 2007;196(Supplement_1):S86S107.

30. Isaakidis P, Cox HS, Varghese B, Montaldo C, Da Silva E, Mansoor H, et al. Ambulatory multi-drug resistant tuberculosis treatment outcomes in a cohort of HIV-infected patients in a slum setting in Mumbai, India. PloS one. 2011;6(12):e28066.

\section{Tables}

Table 1: Baseline characteristics of the MDR-TB patients 
Variable

$\begin{array}{lll}\begin{array}{l}\text { All patients } \\ (n=69)\end{array} & \text { Male } & \text { Female }(n=21) \\ N(\%) & (n=48) & N(\%) \\ & N(\%) & \end{array}$

\section{Age (in years)}

Median (IQR)

$35(27-45)$

$36(28.5-$

$46.5)$

$31(27-45)$

Time to treatment initiation (in days)

Median (IQR)

$$
27.5(6-89) \quad 26(5-74) \quad 43(11-120)
$$

Sex

Female

Male

$\begin{array}{lll}21(30.4 \%) & \text { NA } & \text { NA } \\ 48(69.6 \%) & \text { NA } & \text { NA }\end{array}$

\section{Drug susceptibility resistance profile}

Isoniazid and Rifampicin

Isoniazid, Rifampicin \& other first line

MTB/Rif based on Gene expert result

$\begin{array}{lll}10(14.5 \%) & 6(12.5 \%) & 4(19.0 \%) \\ 20(29.0 \%) & 14(29.2 \%) & 6(28.6 \%) \\ 39(56.5 \%) & 28(58.3 \%) & 11(52.4 \%)\end{array}$

\section{Treatment regime}

Individualized

Standardized

$\begin{array}{lll}3(4.4 \%) & 1(2.1 \%) & 2(9.5 \%) \\ 66(95.6 \%) & 47(97.9 \%) & 19(90.5 \%)\end{array}$

HIV status

Negative

Positive**

$\begin{array}{lll}39(56.5 \%) & 29(60.4 \%) & 10(47.6 \%) \\ 30(44.5 \%) & 19(39.6 \%) & 11(52.4 \%)\end{array}$

ART status of HIV positive cases

On ART

Not on ART

$\begin{array}{lll}27(90 \%) & 17(89.5 \%) & 10(90.9 \%) \\ 3(10 \% & 2(10.5 \%) & 1(9.1 \%)\end{array}$

\section{CD4 Cell count/microliter of blood}

CD4 cell count is known

CD4 cell count is not known

Median CD4 cell count (IQR)

$\begin{array}{lll}28(93.3 \%) & 19(100 \%) & 9(81.8 \%) \\ 2(6.7 \%) & 0(0 \%) & 2(18.2 \%) \\ 206(113- & 268(113-498) & 130(113-174) \\ 364.5) & & \\ 20(29.0 \%) & 16(33.3 \%) & 4(19.1 \%) \\ 37(53.6 \%) & 24(50.0 \%) & 13(61.9 \%) \\ 5(7.3 \%) & 1(2.1 \%) & 4(19.1 \%)\end{array}$

New

\section{TB treatment history/patient type}

Category 1 failure

Category 2 failure 
Relapse

Treatment after loss-to-follow up/defaulter

\section{Current treatment hospital}

Treatment Initiating hospital (RRH)

Follow up health facility

\section{Weight measurement (in kilograms)}

Gained weight: Percent (Mean weight gain, $\mathrm{SD})$

Lost weight: Percent (Mean weight lost, SD)

No change in weight: Percent (Mean, SD)

\section{Adverse events}

Yes

No

Mean (SD) number of adverse events

Range
$1(1.5 \%)$

$6(8.7 \%)$

$21(30.4 \%)$

$35(72.9 \%)$

$13(61.9 \%)$

$48(69.6 \%)$

$13(27.1 \%)$

$\mathrm{n}=40$

$\mathrm{n}=19$

$67.8(4.7, \pm 3.2)$

$65.0(4.9, \pm 3.3)$

$73.7(4.1, \pm 3.1)$

$30.5(2.8, \pm 2.3)$

$0.02(0, N A)$

$32.5(2.5, \pm 2.6)$

$2.5(0, N A)$

$26.3(3.5, \pm$ $0.9)$

0 (NA, NA)

17 (80.9\%)

4 (19.1\%)

$16(23.2 \%)$

$12(25.0 \%)$

1.9 (1.48)

1.8 (1.69)

1.8 (1.79)

$(0.0-7.0)$

$(0.0-5.0)$

** - All HIV positive patients received ART and Cotrimoxazole prophylaxis

Table 2: Frequency of drug reactions and how they were managed 


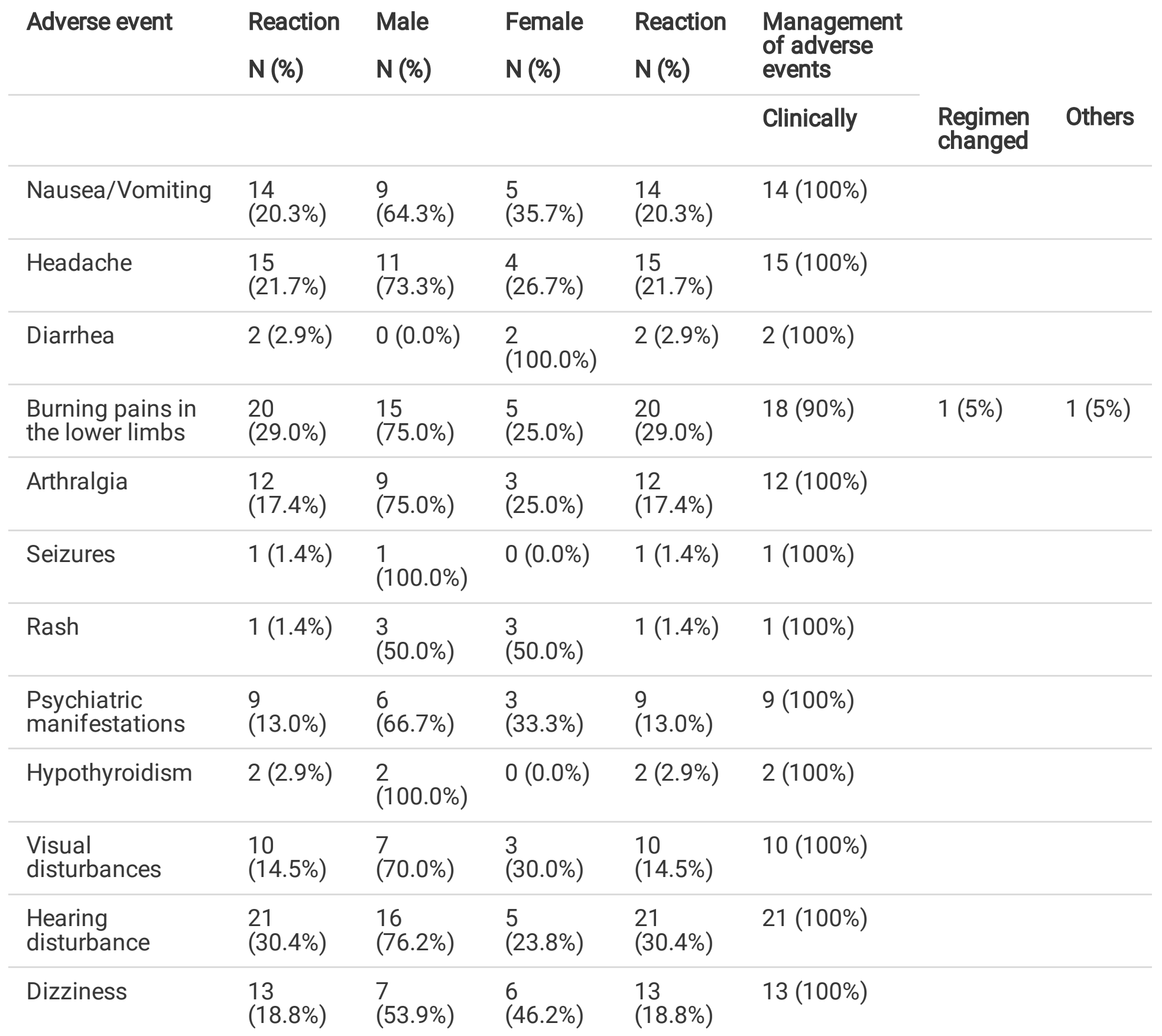

Table 3: Six-months interim treatment outcomes, culture conversion time and adverse events profiles

Page 16/18 
Variable

$\begin{array}{lll}\begin{array}{l}\text { All patients } \\ (n=69)\end{array} & \begin{array}{l}\text { Male } \\ (n=48)\end{array} & \begin{array}{l}\text { Female } \\ (n=21)\end{array} \\ N(\%) & N(\%) & N(\%)\end{array}$

Six months interim treatment outcomes

Favourable outcome

$59(87.0 \%) \quad 41(85.3 \%) \quad 18(85.7 \%)$

Culture converted

Unfavourable outcome

Remained culture positive

$1(1.5 \%) \quad 0(0.0 \%) \quad 1(4.8 \%)$

$3(4.4 \%) \quad 3(6.3 \%) \quad 0(0.0 \%)$

$3(4.4 \%) \quad 1(2.1 \%) \quad 2(9.5 \%)$

$3(4.4 \%) \quad 3(6.3 \%) \quad 0(0.0 \%)$

Culture unknown

Died

Lost-to-follow up

Culture conversion time (by the sixth month of treatment)

$45(76.3 \%) \quad 10(24.4 \%) \quad 4(22.2 \%)$

By two months $14(23.7 \%) \quad 31(75.6 \%) \quad 14(77.8 \%)$

Between three and six months

$\begin{array}{llll}\text { Mean time to culture conversion (SD) } & 1.98( \pm 0.86) & \begin{array}{l}2.07 \\ ( \pm 0.88)\end{array} & 1.78( \pm 0.81)\end{array}$

Table 4. Relationship between interim 6-months treatment outcome and type of patient

\begin{tabular}{|c|c|c|c|c|c|c|}
\hline Type of & $\begin{array}{l}\text { Unfavourable outcome } \\
(\mathrm{N}=10)\end{array}$ & & $\begin{array}{l}\text { Favourable outcome } \\
(\mathrm{N}=59)\end{array}$ & & $\begin{array}{l}\text { Chi- } \\
\text { square }\end{array}$ & Paluo \\
\hline & $\mathbf{N}$ & $\%$ & $\mathbf{N}$ & $\%$ & & \\
\hline $\begin{array}{l}\text { Treatment } \\
\text { failure }\end{array}$ & 6 & $60.0 \%$ & 51 & $86.4 \%$ & 4.2 & $0.04^{\star}$ \\
\hline Defaulter & 2 & $20.0 \%$ & 4 & $6.8 \%$ & 1.9 & 0.17 \\
\hline New & 1 & $10.0 \%$ & 4 & $6.8 \%$ & 0.13 & 0.72 \\
\hline Relapse & 1 & $10.0 \%$ & 0 & $0 \%$ & 6.0 & $0.01 *$ \\
\hline
\end{tabular}

\section{Figures}




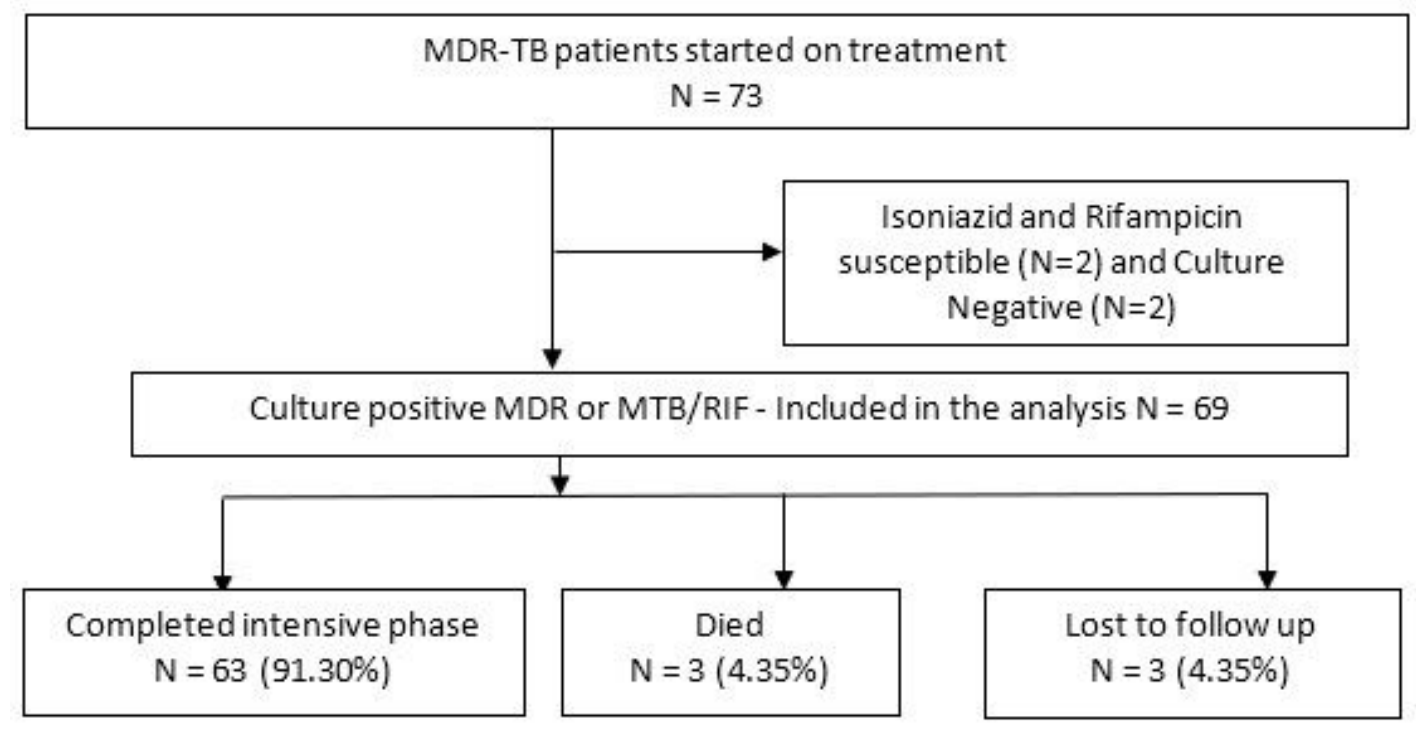

Figure 1

Flow chart for patients initiated on MDR-TB treatment from April 2013 to June 2014

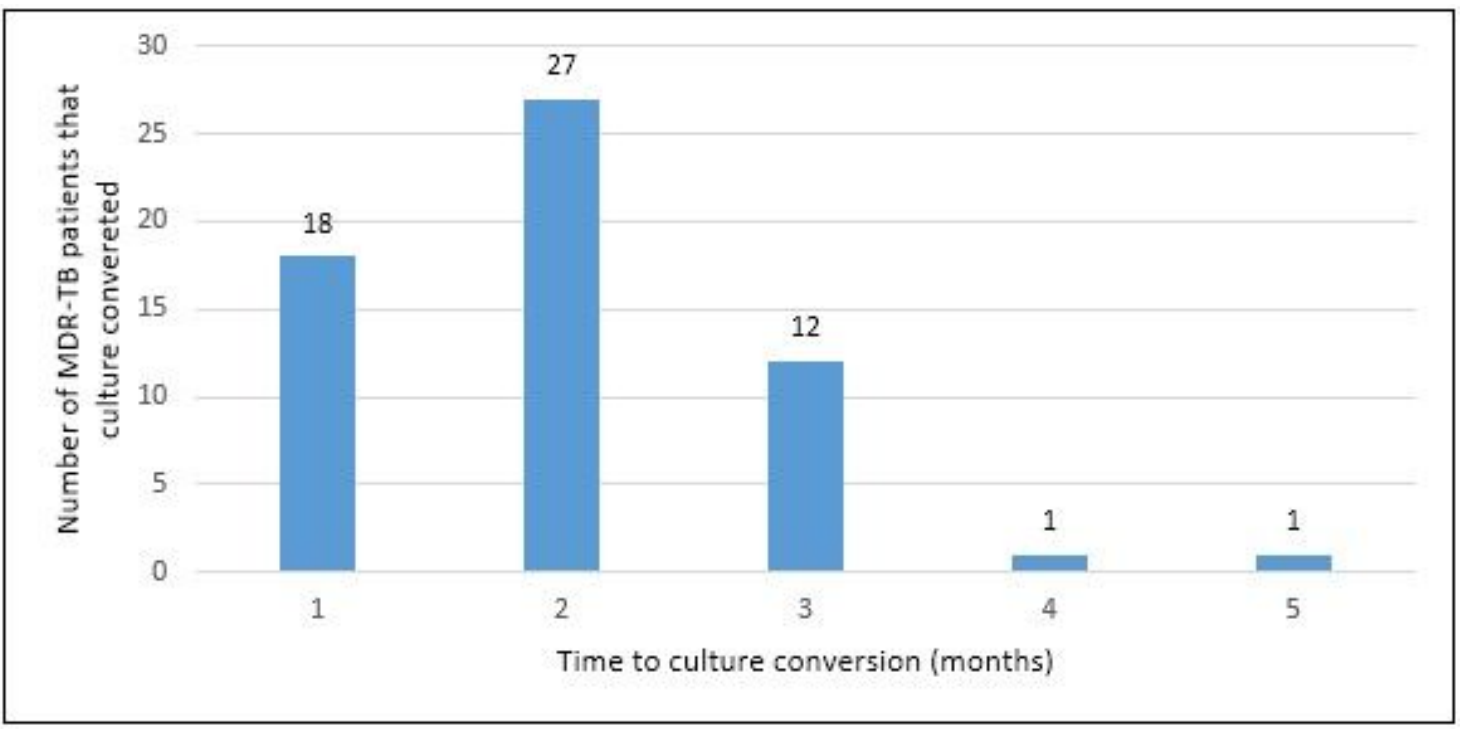

\section{Figure 2}

Time to sputum culture conversion among 59 patients with favorable interim treatment outcomes 